\title{
Evidence-based rehabilitation in forensic psychiatry $^{\dagger}$
}

\author{
PER LINDQVIST and JEREMY SKIPWORTH
}

\section{Background The extent to which forensic psychiatric rehabilitation alters an individual's level of risk is unclear.}

\begin{abstract}
Aims To highlight some essential features of a forensic psychiatric rehabilitation system, and to discuss risk assessment in this context to create a conceptual framework for risk research and practice.
\end{abstract}

\section{Method The applicability of risk assessment instruments to forensic psychiatric rehabilitation was examined. Core processes and elements considered essential in this type of rehabilitative work were reviewed.}

\section{Results Current risk research has limited application to rehabilitation. Future research aimed at analysing forensic psychiatric rehabilitation will be hampered by the complexity of the treatment systems and the number of methodological issues relevant to this type of research.}

\section{Conclusions Novel research approaches are suggested to analyse further the risk factors and processes important in forensic psychiatric rehabilitation.}

\section{Declaration of interest None.}

The assessment of the probability of mentally disordered offenders committing violent and criminal acts continues to be subject to extensive research. If such research is to be applicable and ethically acceptable, it must be grounded in clinically-based studies that link risk assessment to management strategies capable of ameliorating that risk. The profession of forensic psychiatry should not restrict itself to being an entrepreneurial risk assessment industry without ties to the traditional roles of medicine: to treat and to care. Thus, treatment and rehabilitation need to occupy a central position in forensic mental health. A scientific evaluation of our present practice will form a valid base for risk prediction that is sensitive to the dynamics of forensic practice. These studies should utilise both qualitative and quantitative methodologies, and focus on the questions of whether or not, to what extent and when forensic psychiatric rehabilitation alters the individual's level of risk. There is a dearth of literature addressing these issues, which leaves clinical practice without a solid scientific base for its actions. Variables derived from risk research presented hitherto are largely static and disregard the potential for rehabilitation to change the mental state and behaviour of an offender. In losing sight of this central task of forensic mental health they seriously diminish it as a discipline. This paper discusses some essential features of a forensic psychiatric rehabilitation system in an endeavour to create a conceptual framework for research and practice. We suggest that this research paradigm will be supportive of, and conducive to, ongoing developments and advances in rehabilitative practice in forensic mental health.

\section{THE EVOLUTION OF RISK RESEARCH}

Several studies conducted during the 1990 s

†See editorial pp. 307-3II, this issue. increased risk in violent behaviour among people with major mental disorders when compared with the general population (Swanson et al, 1990; Link et al, 1992; Monahan, 1992). One question that arises from such research is in which subgroups of patients and in which contexts does violence by people with mental illnesses most frequently occur. The development of actuarial risk assessment tools has coincided with this second generation of research, and much faith is invested in these instruments from which probability predictions are made. In our minds, this is a hazardous tack on which to embark if not properly safeguarded. These tools are largely based on static and historical factors that cannot hope to be targeted or changed by rehabilitative endeavours.

One risk is that some patients, falsely associated with a high actuarial risk score (false positives), will be locked up because of 'guilt by statistical association'. The equally unacceptable risk is that those patients with a falsely reassuring low actuarial risk score (false negatives) may persuade judges or review panels that they are ready to be released despite clinical concerns. Finally, the correctly identified group with a high probability of future violence is in danger of having the label 'dangerous' attached. This risks their being treated as if they have an immutable quality of viciousness rather than possessing a range of properties and predispositions, many open to modifications.

There is evidence, although disputed, that mental health professionals' predictions of violence are substantially better than chance (Mossman, 1994). It appears that the addition of actuarial risk assessment tools to clinical judgement alone improves the accuracy of determining a patient's risk of recidivism (Borum, 1996; Gardner et al, 1996; Bonta et al, 1998). However, the currently used predictor variables of recidivism are similar in different offender groups, irrespective of any contribution from mental illness, and are often regarded as largely immutable. This does not obviate the task of psychiatric rehabilitation, nor render the treating team jail wardens awaiting the expiry of a sentence to indicate that community placement is appropriate. That forensic psychiatric populations compare favourably with matched criminal populations with respect to recidivism is not surprising, given the vastly disparate nature of their treatment and rehabilitation. Better questions to pose 
then may be what is there to treat, and what is it about forensic psychiatric rehabilitation that works?

\section{FUTURE RISK RESEARCH}

Delineating dynamic risk variables (or treatment factors) is the first step towards investigating the effectiveness of forensic psychiatric rehabilitation. However, such pursuits must avoid overemphasising the reliability of these predictor variables without due consideration of their validity. That is, although a dynamic risk variable translates into a potential focus for treatment, even if its reduction reliably predicts a reduced risk of recidivism, at this point we have not ascertained the validity of this construct in rehabilitation-hence the importance of further clinically-based research.

The need for more and better scientific evaluations of treatment programmes is therefore overdue in the evolution of risk research, because successful interventions to reduce risk will need to be founded on both reliable and valid assumptions. There is an inherent risk in the clinical setting that in viewing the notion of risk assessment purely within a quantitative paradigm based on static and historical factors we will potentially dilute the effectiveness of other rehabilitative endeavours that aim to change the mental state and behaviour of an offender.

\section{DYNAMIC RISK VARIABLES}

Relatively static risk factors such as personal demographics and personality characteristics are uncommon targets for rehabilitation, yet they form the core of all risk assessment tools currently in use. It is predictable that a patient who has a history of persistent antisocial behaviour and falls ill with a major mental disorder will be an ongoing risk to the public even after the mental disorder is controlled. In risk rehabilitation we must focus on risk factors that potentially can be changed.

Risk assessment can be linked to at least four dynamic features of the individual patient and their treatment setting, each with varying resistance to change, and necessitating a different therapeutic task:

(a) the disorder itself, where active psychotic symptoms can create dangerous situations, especially if the person feels threatened, controlled or is commanded to harm others (Link et al, 1992; Wessely et al, 1993; Junginger, 1995, 1996); (b) family problems and poor sociocultural circumstances, including the extent and quality of interactions with family, peers and society;

(c) substance misuse, which can result in impairments of judgement, empathy, temper and impulsiveness, both on the basis of acute intoxication and the sequelae of long-term misuse (Lindqvist, 1986; Lindqvist \& Allebeck, 1989; Swanson et al, 1990);

(d) anti-therapeutic system dynamics creating stigmatised, expelled, underrated, incarcerated or misunderstood mental patients, with reciprocal friction and aggression.

Many forensic patients have multiple and complex diagnoses and are subjected to treatment that varies widely in quality and efficacy. This may be one reason for the dearth of literature on treatment evaluation. What we are to analyse is almost as difficult as capturing the wind. What is evident, however, is that any assessment that looks only at the patient and neglects to analyse the forensic system within which the individual lives will reveal only part of the answer to what will constitute optimal treatment for that individual. In the past, there has been insufficient analysis of those factors external to the patient and of their influence on the prognosis. It is, therefore, essential to focus therapeutic rehabilitative endeavours on factors within both the person and the rehabilitative environment in which they live.

\section{FORENSIC PSYCHIATRIC REHABILITATION}

Rehabilitation is a process where the outcome is the result not only of the sum of individual contributions within the treatment system, but also of the interacting effects of these various efforts. At best these contributions will be synergistic and complementary; at worst, the effect may be negative and associated with a detrimental outcome. These individual contributions or factors are innumerable but we believe that some items preferentially determine the outcome and form the core of a therapeutic culture and process of rehabilitation. We will not address in any detail the specific treatment of positive and negative symptoms, depression, aggression, criminality, life skill deficits and substance misuse because these have been reviewed extensively elsewhere (e.g. Rice \& Harris,
1997). In our view, they are also to an extent subservient to the importance of a therapeutic culture.

\section{Shared values and goals}

Viewing forensic rehabilitation as a process infers that different parts within the forensic setting make their own unique contributions to promoting the health of the individual. The culture of the treatment setting submits patients to the values, ideals and goals prevailing in the system. If this culture is not consistent, trustworthy, competent and caring, a good outcome is jeopardised. Inconsistencies and unpredictability are recurrent features in the personal history of many forensic patients, and consequently dysfunctional system dynamics and diffuse expectations will reinforce previous life experiences. Experiences of unity, collaboration, solid agreements and foresight would be novel for these patients.

\section{Staff continuity}

A system in which the staff come and go will not be able to promote the formation of solid treatment alliances. In our experience, trust is one of the most important foundations upon which rehabilitative work can be built. A trusting relationship takes time to nurture, especially in forensic populations. The ability of the system to provide patients with a broad variety of staff, enabling such trust and alliances to emerge and be sustained, is vital for the long-term effect of treatment. On the other hand, a system where staff never leave their job but for retirement or attrition is most likely counter-productive; like living in a stagnant pool with stale, anaerobic water, only nourishing parasites.

A fresh stream of therapeutic integrity is essential to counteract this process. Staff training and skills-building should mirror rehabilitative work with patients. Staff need to examine their skills base and address any deficits through supervision, education, ongoing monitoring and feedback of their professional growth and performance. Thus, the need to become more actively involved in the process of rehabilitation becomes established in the culture.

Residents in health-promoting rehabilitative settings find themselves in what for some is a strangely supportive and nurturing setting. The fostering of such an environment and culture can allow the patient to build confidence and self-esteem, initially in the context of the 
therapeutic community. Thereafter, these skills can be generalised to contact with the wider community. The personal experience can be brought back to the therapeutic community and, with staff and peer support, re-internalised to enhance the patient's concept of self and of competence.

\section{Timing of the initiation of the rehabilitation process}

It can be hypothesised that the patient's long-term risk will decrease most significantly if rehabilitation commences soon after admission, because this will allow maximal exposure to the rehabilitative process. The therapeutic tasks will change through the course of confinement, and one should not underestimate the magnitude of the initial task of dealing with the legal process when facing serious charges, and the uncertainty that this can create in developing treatment approaches.

Institutional life inevitably counteracts autonomy and self-support. It is not surprising that the most successful rehabilitative endeavours occur in the least restrictive environment (Andrews et al, 1990; Test, 1992). Although legal sanctions and/or individual risk appraisal may necessitate institutional care, it is essential that institutional attitudes, which may be convenient for the staff and the patients, are intensively challenged. The problem with forensic work is seldom to succeed in making the patients adjust to the institution. Rather, the task is to enable them to live peacefully outside an institution in a lessstructured and supervised milieu, with neighbours who are not on the payroll.

Failures of a patient in transition to the community may well be the product of a system failure. Being too briskly exposed to new demands in new settings, and denied access, overnight, to the security and familiarity of the preceding treatment environment will invite failure. For some, the treatment setting and fellow patients will have been their home and family for many years.

\section{Family relationships}

Even though a hospital admission will be a decisive event for any forensic patient, relationships with family members often remain what matters most, for better and for worse.

However, because many forensic patients are severely disturbed with multiple and grave diagnoses and a history of offending, many families are burned out and disillusioned. They may have experienced poorly resourced hospital and community-based psychiatric services and, not unfairly, apportion blame for the offending on these deficits. Forensic services must expect some of this frustration to be voiced before solid alliances can be forged between the patient, the family and the psychiatric service. This will take more than an average effort, but when it happens, in our experience, the process of recovery is commonly greatly enhanced. This, therefore, appears to be a factor of great importance.

\section{Social networking/peers}

The patient's keyworker or nurse, therapist and doctor are important people in the treatment process, but they walk alongside the patient for a limited period of time. Their loyalty is professional, linked to their position and restricted to time and place. The patient has little choice in these alliances. In our experience, the patients are more aware of this than most professionals. Social contacts and friends outside the institution have other, seemingly more genuine, reasons to spend time with the patient. Therefore, personal friends outside the hospital are often at least as important as those who are working on the inside. Who is waiting outside? Who will visit and care? Who will open their door on the day of the first leave and eventually at the time of discharge? Different welcome scenarios will be created by a peer who has managed to leave a life of offending and substance misuse, a fragile but stern and loving grandmother and a criminal mob. Without doubt these different scenarios will be associated with different outcomes.

\section{Process insight}

Most forensic patients view their involuntary commitment/hospital orders as a punishment, a sentence to serve. Some professionals unfortunately share this view. We argue that if the patient is discharged with this opinion unchallenged and unaltered, a good outcome is less likely. Thus, a major task for forensic treatment will be to attack the idea of penance through serving time. The purpose of hospitalisation is secondary prevention, that is, to treat a disorder and keep it under control, resulting in fewer hospital admissions, greater autonomy and a better self-understanding. We teach the patient with diabetes what to eat, to exercise and to medicate with the purpose of reducing the complications of the disorder but also to return responsibility to the patient, increasing his or her competence for self-determination. A forensic patient should, ideally, ask for continuous outpatient care on a voluntary basis at the time of discharge. If this happens the rehabilitation has been successful, and experience suggests that the risk of relapse and recidivism is diminished.

\section{The future}

The hopes and fantasies of the patient about his or her future are probably decisive for recovery. At a stretch one might say that it is not what we actually offer the patient on an everyday basis that works, but what lies ahead, beyond our reach: future events that have not happened and may never happen. It is our belief that we should ask far more frequently "what is the rehabilitative task?" rather than "why did he or she do it", because planning determines to a large degree what we do today, whether we are patients with mental disorders or not. Within that perspective, rehabilitation should deal with the patient's preparations and perceptions of postdischarge life with the ultimate goal of forming a realistic, productive and hopeful future. This future is one in which the patient is safe from real or imagined threat from others and has no desire to self-harm. There is always one part of every patient that wants to live a decent social life in peace with fellow human beings.

\section{RESEARCH ON TREATMENT}

Any research aimed at analysing the effects of forensic psychiatric rehabilitation will be hampered by:

(a) the complexity of forensic treatment systems;

(b) the problems of constructing randomised controlled study designs in respect of patients and treatment systems;

(c) the difficulties in defining and operationalising concepts important in the process of recovery;

(d) the problems associated with having low base rate outcome measures;

(e) incarceration effects, and the effects of ageing;

(f) the inability of current risk assessment tools to assist reliably in rehabilitative work. 
Measuring the post-discharge effect of forensic rehabilitation is fraught with difficulty, whether one works within a quantitative or a qualitative paradigm. However, the description and analysis of the treatment system requires recognition as an important variable in the scientific analysis of outcomes in this area of research. Gottfredson (1984) proposed programme development evaluation as a way of measuring the quality and internal integrity of interventions, and this approach has been usefully applied to forensic treatment settings (Rice et al, 1990).

Another way to circumvent the temptation to launch reductionistic studies that focus on one easily-measured item at a time would be to organise a number of separate forensic psychiatry services into a joint collaboration, creating a situation that resembles a natural experiment. The legal and professional practices vary within and between countries and states, whereas in our experience patient characteristics and the nature of offences do not know national borders. With this design, pre-admission data, treatment factors and post-discharge information could be collected and configured uniformly at several sites simultaneously, creating the possibility for comparative research. Such a collaborative approach would allow both qualitative and quantitative research endeavours, leading to the development of more valid and reliable measures of prognostic significance in rehabilitation with these populations. Hopefully, this will capture some core elements of good forensic psychiatric practice, which will enable us to throw away some bad practices and begin to create a more solid scientific, evidence-based practice in forensic psychiatric rehabilitation.

\section{ACKNOWLEDGEMENT}

We thank Frank Tracey, who is, in a way, the instigator of this paper. He has provided us with the inspiration and practical grounding upon which many of the ideas in this paper are based, and made valuable comments on earlier versions of the manuscript.

\section{REFERENCES}

Andrews, D. A., Zinger, I., Hodge, R. D., et al (1990) Does correctional treatment work? A clinically relevant and psychologically informed meta-analysis. Criminology, 28, 369-404.

Bonta, J., Law, M. \& Hanson, K. (1998) The prediction of criminal and violent recidivism among

\section{CLINICAL IMPLICATIONS}

- The scientific evaluation of rehabilitating offenders with mental disorders is in its infancy, and currently available risk assessment instruments have limited applicability in guiding this process.

- The rehabilitative focus on patient factors as indicators of progress neglects the identification and development of system factors responsible for the patient's progress.

- Novel research strategies that analyse both patient and treatment factors are needed, so that an evidence-based approach to this work can be established.

\section{LIMITATIONS}

- The dearth of research into rehabilitation programmes for offenders with mental disorders leads to speculative assumptions and opinions requiring further evaluation.

- Assessing the utility of different treatment approaches for specific needs relevant to risk management was beyond the scope of this article.

- Not all of the potential core issues in rehabilitation were addressed.

PER LINDQVIST, PhD, Örebro Forensic Psychiatry Service, Eken, Örebro, Sweden; JEREMY SKIPWORTH, FRANZCP, Regional Forensic Psychiatry Service, Auckland, New Zealand

Correspondence: Per Lindqvist, Director, Örebro Forensic Psychiatry Service, Eken, S-70I85 Örebro, Sweden. e-mail: per.lindqvist@orebroll.se

(First received 19 May 1999, final revision 29 November 1999, accepted 7 December 1999)

mentally disordered offenders: a meta-analysis. Psychological Bulletin, 123, 123-142.

Borum, R. (1996) Improving the clinical practice of violence risk assessment. Technology, guidelines and training. American Psychologist, 5I, 945-956.

Gardner, W., Lidz, C. W., Mulvey, E. P., et al (1996) Clinical versus actuarial predictions of violence in patients with mental illness. Journal of Consulting and Clinical Psychology, 64, 602-609.

Gottfredson, G. D. (1984) A theory-ridden approach to program evaluation: a method for stimulating researcher-implementer collaboration. American Psychologist, 39, $1101-1112$

Junginger, J. (1995) Command hallucinations and the prediction of dangerousness. Psychiatric Services, 46 9|1-9|4.

_ (1996) Psychosis and violence: the case for a content analysis of psychotic experience. Schizophrenia Bulletin, 22, $91-103$

Lindqvist, P. (1986) Criminal homicide in northern Sweden, 1970-1981: alcohol intoxication, alcohol abuse and mental disease. International Journal of Law and Psychiatry, 8, 19-37.

\& Allebeck, P. (1989) Schizophrenia and assaultive behaviour: the role of alcohol and drug abuse. Acto Psychiatrica Scandinavica, 82, 191-195.
Link, B. G., Andrews, H. \& Cullen, F. T. (1992) The violent and illegal behaviour of mental patients reconsidered. American Sociological Review, 57, 275-292.

Monahan, J. (1992) Mental disorder and violent behaviour. American Psychologist, 47, 5II-52I.

Mossman, D. (1994) Assessing predictions of violence. Being accurate about accuracy. Journal of Consulting and Clinical Psychology, 62, 783-792.

Rice, M. E., Harris, G. T., Quinsey, V. L., et al (1990) Planning treatment programs in secure psychiatric facilities. In Law and Mental Health. International Perspectives, vol. 5 (ed. D. N.Weisstub), pp. 162-230. New York: Pergamon

_ \& _ (1997) Treatment of mentally disordered offenders. Psychology, Public Policy, and Law, 3, 126-183.

Swanson, J.W., Holzer, C. E., Ganju, V. K., et al (1990) Violence and psychiatric disorder in the community: evidence from the epidemiologic catchment area surveys. Hospital and Community Psychiatry, 41, 76I-770.

Test, M. A. (1992) Training in community living. In Handbook of Psychiatric Rehabilitation (ed. R. Liberman), pp. 153-170. New York: Macmillan.

Wessely, S., Buchanan, A., Reed, A., et al (1993) Acting on delusions. I: Prevalence. British Journal of Psychiatry, 163, 69-76. 